gen diese Regionen einen bedeutenden wirtschaftlichen Substanzverlust, für deren vollständigen Ersatz die in der jeweiligen Region vorhandenen endogenen Entwicklungspotentiale nicht genügen dürften. ${ }^{6} \mathrm{Ge}$ wisse Entwicklungsmöglichkeiten bieten sich allenfalls im Wohnbereich für Regionen, welche in Pendeldistanz zu einem Großzentrum liegen, sowie an erstklassigen Tourismusstandorten, die sich im verschärften internationalen Wettbewerb am ehesten zu behaupten vermögen.

Der Schluß liegt nahe, daß die wirtschaftliche Polarisierung zwischen den starken Zentrenregionen und den strukturell benachteiligten Randregionen in der Schweiz in den nächsten Jahren nicht zuletzt aufgrund unterschiedlicher Entwicklungschancen des Finanzplatzes Schweiz und des Werkplatzes Schweiz deutlich zunehmen wird. Der Wettbewerb zwischen dem Werkplatz und dem Finanzplatz scheint damit nicht nur für die Schweiz als Ganzes sowie für die wirtschaftliche und politische Integration der Schweiz in Europa, sondern auch für die Entwicklung in den verschiedenen Regionen eine Schicksalsfrage zu sein.

\section{Anmerkungen}

' BHP - BRUGGER, HANSER und Partner (1991): EG 92 Neue Anforderungen an die Regionalpolitik? Schriftenreihe Biga, Beiträge zur Regionalpolitik Nr. 2, Bern.

${ }^{2} z$ z.B. WORLD COMPETIVIVENESS REPORT (1995), in: "NZZ" vom 6.12.1995, S. 21; PORTER, M. E. (1990): Nationale Wettbewerbsvorteile. Erfolgreich konkurrieren auf dem Weltmarkt; NAVARINI, E.von (1995): Direktinvestitionen ausländischer Unternehmen in der Schweiz, in: BUNDESAMT FÜR KONJUNKTURFRAGEN (1995): Wirtschaftliche Auswirkungen des EWR-Neins, Band 2, Bern.

${ }^{3}$ HANSER, Ch. / CAVELTI, G. (1995): Strukturbericht Kanton Thurgau: Stärken, Schwächen und Perspektiven der Wirtschaft im Kanton Thurgau, Zürich.

${ }^{4}$ HAUSER, H. / BRADKE, S. (1991): EWR-Vertrag, EG-Beitritt, Alleingang: Wirtschaftliche Konsequenzen für die Schweiz. Zürich/Chur; RISTRA, R. (1995): The consequences of foreign trade, in:BUNDESAMT FÜRKONJUNKTURFRAGEN (1995): Wirtschaftliche Auswirkungen des EWR-Neins, Band 2, Bern.

${ }^{5}$ HANSER, Ch., et al. (1996): Ticino 2000: Struktur und Entwicklung des Handels- und Finanzplatzes Tessin (erscheint im Herbst 1996).

${ }^{6}$ HANSER, Ch. / KUSTER, J. (1993): Grundzüge der Raumordnung Schweiz, Überprüfung der Hauptstrategien für den Siedlungsraum, Zürich.

\title{
Le paradoxe des régions en déclin
}

Depuis peu, certains cantons romands font l'objet d'un paradoxe économique lié à la coexistence d'un déclin économique visible au niveau statistique et de l'émergence de "clusters" d'activités dynamiques, qui attirent un nombre croissant de sociétés étrangères d'envergure mondiale. A partir de ce paradoxe, le présent article entend montrer que les indicateurs habituellement utilisés pour évaluer le dynamismeéconomique des régions ne reflètent pas forcément la réalité. En effet, ces indicateurs conventionnels considèrent les résultats de la dynamique économique passée, au lieu de prendre en compte les déterminants de la dynamique économique émergente, incorporés dans la compétitivité structurelle de systèmes de production régionaux. De la sorte, on préjuge de l'avenir d'une région sur ce qu'elle a été et non pas sur ce qu'elle peut devenir, en risquant notamment d'orienter la politique économique et technologique vers de fausses cibles. Il y a donc un enjeu certain à intégrer les facteurs de compétitivité structurelle dans l'évaluation du dynamisme économique régionale, même si à l'heure actuelle on ne trouve pas de statistiques permettant la mesure de tels facteurs d'ordre qualitatifs. Cela permettrait d'identifier l'émergence de systèmes productifs d'avenir et d'en tenir compte dans les politiques de développement économique régional.
Les cas examinés dans cet article se réfèrent aux cantons de Genève, de Vaud, de Fribourg et de Neuchâtel, dans la mesure où ces zones illustrent bien le paradoxe du déclin économique doublé d'une progression de l'attractivité économique. La première section met en évidence le déclin présumé de ces cantons, compte tenu de leur évolution récente par rapport à la moyenne nationale en termes de chômage et de produit intérieur brut réel. La deuxième section aborde la question de l'émergence de "clusters d'activités» et des implantations de sociétés étrangères dans les régions analysées, en précisant les raisons majeures qui ont dicté ces choix de localisation. Enfin, la dernière section esquisse les lignes directrices d'une nouvelle démarche d'analyse du dynamisme régional prenant en compte la compétitivité structurelle des régions.

Denis Maillat, Prof., Giannicola Ballin, Florian Németi, Institut de recherches économiques et régionales (IRER), Université de Neuchâtel, 7, Pierre-à-Mazel, 2000 Neuchâtel 


\section{Mesure conventionnelle du dynamisme régional: le cas de la Suisse romande}

Le déclin économique régional possède une indéniable connotation de perspective à long terme. Pour les régions, il signifie une menace sérieuse de dégradation de leur capacité durable à produire des biens et des services rémunérateurs et créateurs d'emplois. Pourtant, cette notion prévisionnelle n'est habituellement pas évaluée ex ante, mais ex post, à travers l'évolution récente de variables telles que le taux de chômage, le revenu brut par habitant, le coefficient de spécialisation sectorielle', la capacité financière ou encore la productivité du travail. Cette démarche d'analyse classique repose sur l'hypothèse implicite que les perspectives de développement économique d'une région se situent dans le prolongement immédiat de tendances observées au cours d'un passé plus ou moins récent. En vertu de cette hypothèse, une région considérée comme déclinante ne devrait donc pas, a priori, présenter d'attraits pour des entreprises dynamiques externes à la région et à la recherche d'un lieu d'implantation. Or, plusieurs cas observés récemment en Suisse romande - sur lesquels nous reviendrons ultérieurement - contredisent cette logique.

La prise en compte de l'évolution du taux de chômage (mesuré sur les emplois à plein temps) des cantons suisses entre janvier 1990 et décembre 1995 montre que les cantons romands se situent bien au-dessus de la moyenne nationale (figure 1). Seul le Tessin, dont le taux de chômage en janvier 1996 était de 8,5\%, est encore plus mal loti que les cantons romands. Les statistiques de l'OFIAMT'2 pour janvier 1996 font ressortir des écarts marqués par rapport à la moyenne nationale:

Tableau 1 taux de chômage cantonaux en janvier 1996

\begin{tabular}{lrrrrrrr}
\hline Région & $\mathrm{CH}$ & $\mathrm{FR}$ & $\mathrm{NE}$ & $\mathrm{JU}$ & $\mathrm{GE}$ & VD & VS \\
Chômage (\%) & 4,5 & 5,2 & 5,8 & 6,1 & 6,9 & 7,3 & 7,7 \\
Ecart & - & $+0,7$ & $+1,3$ & $+1,6$ & $+2,4$ & $+2,8$ & $+3,2$ \\
\hline
\end{tabular}

Source: OFIAMT, 1996
Le chômage observé au cours d'une période de moyenne durée ne saurait être le seul critère de déclin pour une région. GAUDARD ${ }^{3}$ établit une typologie des régions comportant quatre cas de figure (cantons forts et prospères; cantons forts, mais en déclin; cantons faibles, mais en rattrapage et cantons faibles et en déclin) selon l'état de deux variables: le revenu cantonal par habitant pour une année $\mathrm{t}_{0}$ et la croissance annuelle du revenu cantonal en $\%$, pendant la période allant de $t_{0}$ à $t_{n}$. En utilisant cette typologie, l'Office fédéral de la statistique constate qu'en 1993, les cantons de Neuchâtel, du Jura et de Genève figurent au rang des régions en déclin, alors que les cantons de Fribourg, du Valais et de Vaud apparaissent comme des régions en phase de rattrapage. ${ }^{4}$

Le produit intérieur brut (PIB) par canton peut également servir d'indicateur. Le Valais et Neuchâtel enregistrent un résultat supérieur à la moyenne nationale. Mais seul le Valais figure dans la partie supérieure de la hiérarchie cantonale (1le rang), les autres cantons romands étant situés au-delà du $13 \mathrm{e}$ rang:

Tableau 2 évolution du produit intérieur brut réel entre 1990 et 1993

\begin{tabular}{lrrrrrrr}
\hline Région & VS & NE & CH & FR & $J U$ & VD & GE \\
PIB (1990-93) & $+0,4$ & $-0,3$ & $-0,4$ & $-0,4$ & $-0,7$ & $-1,3$ & $-2,1$ \\
Rang & 11 & 16 & & 18 & 20 & 24 & 26 \\
\hline
\end{tabular}

Source: BAK, 1994

\section{Emergence de «clusters» d'activités dans des régions présumées en déclin}

Le déclin d'une région provient essentiellement d'une perte d'efficacité de son système productif, doublée d'une incapacité de ce dernier à générer de nouvelles sources de valeur ajoutée. Seule une recomposition des activités du système productif vers des produits totalement nouveaux, à partir de savoir-faire traditionnels, peut alors inverser la tendance déclinante. A cet égard,

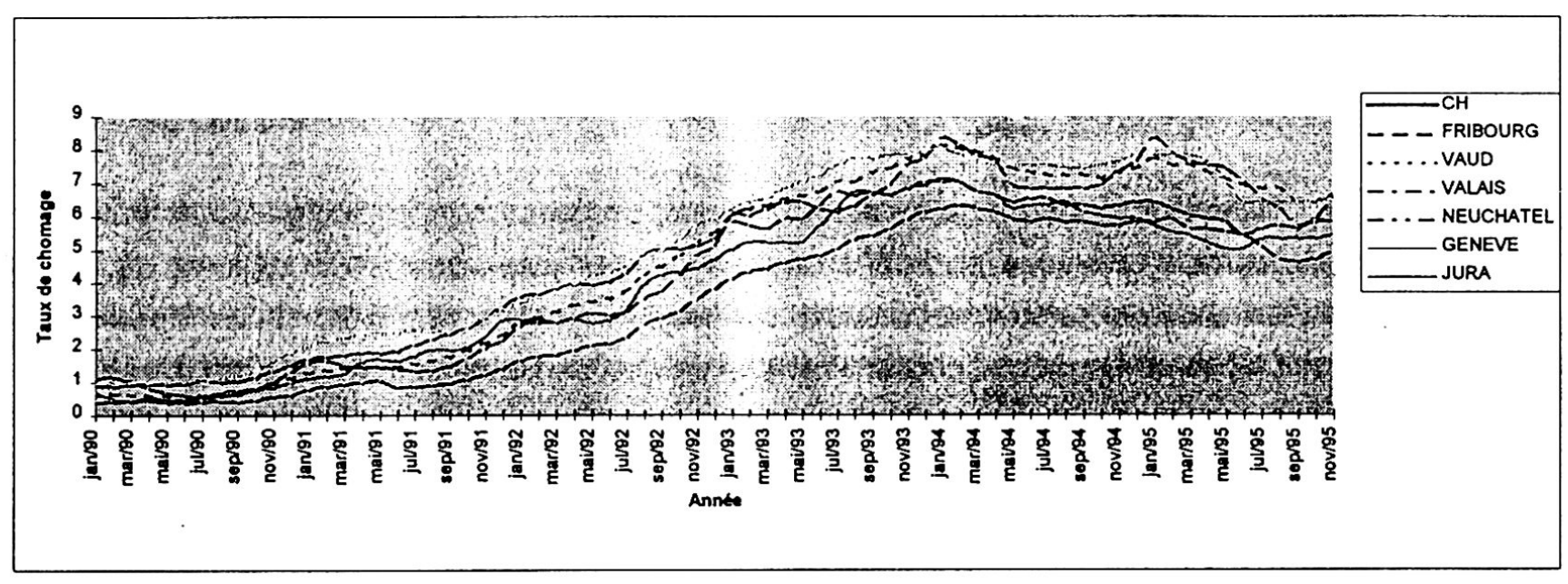

Fig.1 Taux de chômage cantonaux 1990-1995. Source: OFS 
l'exemple de la recomposition partielle des activités horlogères de l'Arc jurassien vers des activités de type microtechnique est significatif. ${ }^{5}$ Selon NÉMETI et PFISTER (1994), cette recomposition est liée à l'émergence de nouveaux réseaux de production et d'innovation, aussi dénommés «clusters» d'activités. MAILLAT et al. définissent un "cluster» d'activités comme un ensemble de réseaux relationnels entre firmes implantées dans une région et à l'intérieur desquels circulent des produits, des services, des informations pertinentes, en termes commerciaux, de management de $R \& D$, etc. Ces informations sont directement exploitables par les membres des clusters.

Compte tenu du fait que ces «clusters» d'activités disposent souvent d'avantages compétitifs au niveau international (PORTER 1990), ils génèrent une forte valeur ajoutée et contribuent à la création de nombreux emplois. Leur apparition semble a priori improbable dans des régions déclinantes. Or, il est fort intéressant de constater que des régions supposées en déclin comme Neuchâtel et le Jura parviennent tout de même à constituer des «clusters» d'activités dans des domaines aussi porteurs que les microtechniques, le domaine médical ou les systèmes de traitement de l'information (HILTPOLD 1995).

\subsection{Exemples d'interaction entre "clusters" d'activités régionaux et implantations de firmes étrangères}

Depuis la fin des années quatre-vingts, des "clusters" d'activités régionaux apparaissent à Neuchâtel (domaine des microtechniques et du médical), à Genève (domaine des télécommunications) et dans la région lausannoise (domaine des biotechnologies). Ils regroupent déjà quelques centaines de sociétés, parmi lesquelles des multinationales en pleine expansion technologique et commerciale. Il s'agit, entre autres, des firmes AT \& T, Sun et IBM à Genève, de Medtronic près de Lausanne et de Silicon Graphics, Baxter, Quantum, Autodesk, Medos, Johnson \& Johnson, Lipomatrix ou encore Mary Kay à Neuchâtel. Il importe de préciser que l'implantation de sociétés étrangères n'est pas à l'origine de ces "clusters» d'activités, mais qu'elle en est la conséquence. Toutefois, il est indéniable que l'arrivée de grandes firmes multinationales a permis la consolidation de "clusters» qui se situaient en phase de démarrage.

Ces sociétés manifestent la volonté de développer une implantation durable, puisqu'elles ont déjà créé plusieurs centaines d'emplois à forte valeur ajoutée et investi plusieurs dizaines de millions de francs. De plus, la présence de ces firmes provoque déjà un effet d'entraînement commercial et technologique sur leurs partenaires régionaux au sein des «clusters» d'activités. ${ }^{6}$ Ceux-ci emploient désormais plusieurs milliers de personnes et génèrent au niveau régional un chiffre d'affaires de plusieurs centaines de millions de francs.? Ils représentent donc un véritable indice de recomposition des activités de systemes productifs régionaux.

Le regain de dynamisme économique régional qu'amènent ces "clusters» d'activités suggère l'hypothèse selon laquelle une région en déclin peut, malgré tout, posséder des atouts structurels. Ces derniers ne ressortent pas des analyses classiques du dynamisme régional. Pourtant, l'implantation de multinationales étrangères laisse supposer qu'ils sont perceptibles lorsqu'on analyse le potentiel de dynamisme d'une région en vue de s'y implanter. Sous un angle prospectif, une région en déclin peut s'avérer attractive.

L'attractivité d'une région ne peut s'expliquer par le seul fait d'une promotion économique agressive, notamment basée sur l'octroi de facilités financières (exonération fiscale, prise en charge d'intérêts, etc.) ou d'infrastructure (mise à disposition de bâtiments, par exemple) car la concurrence internationale régnant désormais en la matière réduit l'importance de ce genre d'avantages. Une région attractive l'est avant tout grâce à des d'avantages compétitifs structurels ${ }^{8}$ - liés à l'environnement industriel, technique et institutionnel régional - sous-estimés ou ignorés par les indicateurs conventionnels du dynamisme économique régional.

\subsection{Motifs d'implantation}

Une partie des avantages compétitifs mentionnés par les firmes qui se sont implantées en Suisse romande n'est pas spécifique à ces cantons, mais plutôt à la Suisse en général. Il s'agit, par exemple, de la qualité de vie, de la sécurité, des infrastructures de transports, de l'offre de formation, de la paix du travail, du niveau des taux d'intérêts ou encore de la stabilité politique. Toutefois, d'autres facteurs propres à la structure économique et institutionnelle des cantons ont aussi joué un grand rôle.

Il s'agit en particulier d'éléments déterminants pour l'évolution concurrentielle sectorielle à moyen et long terme:

- proximité avec des pôles de R \& D à la pointe du progrès;

- présence de nombreux fournisseurs de composants et d'équipements spécialisés;

- disponibilité de main-d'œuvre qualifiée et d'infrastructures de perfectionnement;

- proximité avec des clients exigeants susceptibles d'orienter l'innovation de produits;

- présence de concurrents importants;

- efforts des pouvoirs publics régionaux en matière de transfert technologique entre la science et l'industrie.

\section{Vers une nouvelle approche du dynamisme régional}

L'implantation, dans des régions présumées en déclin, de firmes dynamiques appartenant à des secteurs industriels en forte croissance au niveau international montre les limites de l'approche classique du dynamisme régional. Il convient de la compléter par une nouvelle approche, capable de détecter les foyers de diversification et de reconversion à partir desquels une région peut suppléer au déclin présumé de ses activités traditionnelles. 
Concrètement, on pourrait esquisser une démarche en trois phases à réaliser pour chaque région:

- établir une liste des activités tendant à progresser (sur la base de critères chiffrés, rapportés à la moyenne nationale),

- évaluer l'état et l'évolution récente des facteurs qualitatifs de compétitivité structurelle des domaines d'activités en progression,

- évaluer les potentiels de croissance sur les marchés internationaux des activités régionales dont la compétitivité structurelle est forte.

Les critères à retenir pour mesurer le dynamisme des activités régionales pourraient être l'évolution récente des emplois, de la valeur ajoutée, des savoir-faire, des exportations, de la création d'entreprises, de l'apparition de nouveaux produits/procédés et des dépenses de R \& D. Une fois repérées les activités en progression, il s'agit de vérifier que leurs exigences de développement technique, économique et socioprofessionnel puissent être remplies par les conditions-cadres régionales. Comme il n'existe aucune banque de données nationale ou régionale à ce sujet, seuls des questionnaires périodiques peuvent apporter les informations nécessaires.

Il est évident que cette esquisse de démarche ne constitue qu'un premier pas dans la direction d'une analyse affinée du dynamisme économique des régions par l'approche de la compétitivité structurelle des systèmes de production régionaux. Un travail considérable de formalisation reste à effectuer. Par ailleurs, en l'état actuel des statistiques régionales et sectorielles disponibles, il est encore très difficile, pour ne pas dire impossible, d'approfondir certains aspects quantitatifs et qualitatifs de la démarche que nous suggérons. Cette remarque touche particulièrement les indicateurs ayant trait aux exportations, à la valeur ajoutée et aux nouveaux produits/procédés, ainsi qu'à la mesure de l'évolution structurelle et organisationnelle des chaînes de création de valeur ajoutée au sein des systèmes de production régionaux.

Néanmoins, cette démarche nous paraît digne d'intérêt dans la mesure où la globalisation croissante de l'économie risque de créer des disparités régionales toujours plus grandes et toujours plus changeantes, sans doute au profit des régions qui sauront identifier, exploiter et développer à temps la compétitivité structurelle des systèmes de production susceptibles de progresser. Dans ce contexte, il est donc fort regrettable que l'on ne puisse pas suffisamment intégrer cet ensemble de variables dans l'évaluation du dynamisme économique régional. En effet, tant que l'approche traditionnelle ne pourra pas être dépassée, les régions en déclin risqueront de ne pas être celles que l'on croit.

\section{Notes}

' Une région fortement spécialisée en termes d'emplois et de revenus dans une ou quelques activités économiques en régression connaît une très forte probabilité de décliner.

${ }^{2}$ Office fédéral de l'industrie, des arts et métiers et du travail.

${ }^{3}$ Voir Office fédéral de la statistique (1995), p. 136.

${ }^{4}$ Voir Office fédéral de la statistique (1995), pp. 136-137.

${ }^{5}$ Voir l'étude de Crevoisier (1993) sur les milieux innovateurs de l'Arc jurassien.

${ }^{6}$ Selon les études de Maillat (1993), Forster et Jeanrenaud (1994) et de Vodoz et al. (1993).

${ }^{7}$ Voir Hiltpold (1995b)

${ }^{8}$ Porter (1990) présente ces facteurs dans son "diamant» de compétitivité. Celui-ci explique la compétitivité d'industries concentrées au niveau régional par l'existence à proximité des firmes d'une demande exigeante et d'une infrastructure scientifique de pointe, par l'existence de relations de collaboration au sein des chaînes de valeur ajoutée, de même que par l'existence d'une concurrence régionale forte et peu protégée par les pouvoirs publics.

\section{Bibliographie}

CREVOISIER, O. (1993): Industrie et région: Les milieux innovateurs de l'Arc jurassien, coll. IRER, édi. EDES, Neuchâtel.

FORSTER, S., JEANRENAUD, C. (1993): La promotion économique neuchâteloise: Bilan 93, coll. IRER, édi. EDES, Neuchâtel.

HILTPOLD, P. (1995a): Les clusters, communication présentée à la Table-Top Exhibition de la Chambre Neuchâteloise de Commerce et de l'Industrie et de la Chambre de Commerce et d'Industrie du Jura, 20 février 1996, St-Aubin.

HILTPOLD, P. (1995b): Entreprises actives dans le marché médical, communication présentée à la Table-Top Exhibition de la Chambre Neuchâteloise de Commerce et de l'Industrie et de la Chambre de Commerce et d'industrie du Jura, St-Aubin.

MAILLAT, D. (1993): “Neuchâtel Up» pour une stratégie globale de développement économique, Rapport de travail à l'intention du Conseil d'Etat neuchâtelois.

MAILLAT, D., NÉMETI, F., PFISTER, M. (1992): Système territorial de production et avantages compétitifs: Le cas de l'industrie des microtechniques, Working Papers de I'IRER, Université de Neuchâtel.

NÉMETI, F., PFISTER, M. (1994): Aspects de la compétitivité de l'industrie des microtechniques suisses, coll. IRER, édi. EDES, Neuchâtel.

OFFICE FÉDÉRAL DE LASTATISTIQUE (1995): Annuaire statistique de la Suisse 1996, Verlag Neue Zürcher Zeitung, Zurich.

PORTER, M. E. (1990): The Competitive Advantage of Nations, London, McMillan.

VODOZ, L., ALBERTON, S., REY, M. (1993): La politique valaisanne de promotion économique: une évaluation privilégiant le point de vue des acteurs. Rapport final, CEAT, Lausanne. 